\title{
Considerations Towards Coordinated Control of DFIG-based Wind Farms
}

\author{
Nilanjan Ray Chaudhuri, Member, IEEE, Balarko Chaudhuri, Senior Member, IEEE
}

\begin{abstract}
This paper highlights the important considerations towards coordinated control of doubly-fed induction generator (DFIG) based wind farms for power oscillation damping. The effect of replacing one existing synchronous generator (SG) with power system stabilizer (PSS) by a DFIG on the local mode and also the mode-shapes of the critical inter-area modes is analyzed. With almost zero participation from the DFIG mechanical side and very little observability of low frequency oscillatory modes in signals locally available at the wind farms, choice of appropriate remote feedback signals is discussed. Relative controllabilities of the DFIG rotor current components are compared to determine the most effective control structure. A coordinated control design approach aimed at damping multiple oscillatory modes through more than one DFIG-based wind farms is demonstrated. Heuristic optimization is used for the design problem which is otherwise difficult to solve using an analytical approach. Modal analysis and non-linear simulation results are presented to substantiate the findings.
\end{abstract}

Index Terms-Wind farm, Doubly-fed induction generator (DFIG), Power oscillation damping (POD), Coordinated control.

\section{LisT OF ABBREVIATIONS AND NOTATIONS}

$\begin{array}{ll}\omega_{r} & \text { DFIG rotor angular speed } \\ \omega_{e l} & \text { base electrical speed, 377 rad/s } \\ \theta_{t w} & \text { angle of twist in turbine shaft } \\ k_{s h} & \text { shaft stiffness } \\ c_{s h} & \text { damping coefficient } \\ H_{g} & \text { DFIG inertia } \\ T_{e} & \text { DFIG electrical torque } \\ \psi_{s} & \text { DFIG stator flux } \\ \psi_{q s}^{\prime} & q \text {-axis DFIG stator flux } \\ L_{s s}, L_{r r}, L_{m} & \text { DFIG stator, rotor and mutual inductance } \\ i_{d r}^{\prime} / i_{q r}^{\prime} & d / q \text {-axis DFIG rotor current } \\ i_{d s}^{\prime} / i_{q s}^{\prime} & d / q \text {-axis DFIG stator current } \\ v_{d r}^{\prime} / v_{q r}^{\prime} & d / q \text {-axis rotor voltage of DFIG } \\ R_{s} / R_{r} & \text { DFIG stator/rotor resistance } \\ s_{l} & \text { rotor slip } \\ K_{o p t} & \text { co-efficient of maximum power extraction }\end{array}$

\section{INTRODUCTION}

$\mathbf{S}$ UBSTANTIAL penetration of wind energy, mostly through doubly-fed induction generators (DFIG), would

Support from the EPSRC UK (EP/F029128/1) is gratefully acknowledged.

N. R. Chaudhuri and B. Chaudhuri are with the Control and Power Research group, Imperial College London, London, UK (e-mail: n.chaudhuri@imperial.ac.uk, b.chaudhuri@imperial.ac.uk). potentially change the dynamic behavior of future power systems. System support features, like power oscillation damping, frequency regulation etc., that are usually demanded from conventional synchronous generators (SG), would also be increasingly applicable for wind farms. It is therefore, critical to develop a system level understanding of the influence of wind generators and identify factors and ways of controlling them in a coordinated fashion. Of particular, although not only, interest is the power oscillation damping problem through wind farms which is the focus of this paper.

Several researchers have studied the effect of wind farms on the small-signal stability of AC systems [1], [2], [3], [4], [5]. In [1], [6] typical operational scenarios were considered where a fixed system load was supplied by different relative share of SG and DFIGs. Damping contribution of DFIGs when compared to SGs was found to be higher or lower depending on the operating condition [1]. In [6], DFIG was always found to contribute positively to system damping. Some of the above papers have further explored power oscillation damping through DFIG-based wind farms [6], [2], [3].

In [1], [7], lack of participation of DFIGs in oscillatory modes was highlighted which is substantiated in this paper in terms of the effect on the local modes and also the mode shapes of the critical inter-area modes. Moreover, lack of adequate observability of the critical inter-area modes in signals locally available at the wind farms is demonstrated. In [6], the flux magnitude and angle control (FMAC) approach was used to facilitate network support which is different from the standard vector control technique widely used by the wind farm manufacturers. In the FMAC technique, the traditional fast acting current control loops are not used and thus, the current limiting strategies for the inverters are compromised. As a result, the mechanical side of the DFIG is not 'decoupled' from its electrical side allowing use of local signals. Nonetheless, the potential effectiveness, which turns out to be much more for DFIG than an equivalent SG, of remote feedback signals was not explored. Also the relative controllabilities of the available control variables were not properly assessed from a system theoretic point of view. In this paper the above aspects are taken into consideration in a systematic way.

In [6] and [2], the PSS design for DFIG is based on the fundamental concept of damping torque which is enlightening and works fine for individual wind farms. However, for multiple inter-area modes required to be controlled through multiple wind farms, a system level approach is required rather than looking at individual wind farms to avoid possible adverse interactions and ensure optimal control effort. In this paper a coordinated approach to power oscillation damping control 
design for more than one wind farms is adopted to damp multiple critical inter-area modes.

To understand the system impact of wind farm integration, the first case study considered replacing a single SG with PSS by an equivalent DFIG-based wind generator. Choice of appropriate control structure (i.e. control variables and feedback signals) is demonstrated with a view to match the system dynamic performance in presence of the SG with PSS. Multiple DFIGs were introduced for the second case study to illustrate the choice of appropriate control loops and coordinated design of several wind farm controllers to damp multiple critical inter-area modes. Modal analysis and non-linear simulations results from Matlab/SIMULINK are presented for both the case studies to substantiate the findings.

The contributions of this paper are:

1) Study the impact of replacing conventional synchronous generators (SGs) with PSS by equivalent DFIG-based wind farms on the local and inter-area modes.

2) Identify the problem with damping control through wind farms using locally available signals and present a systematic approach for appropriate control loop (i.e. feedback signals and control variables) selection through modal analysis.

3) For the first time, demonstrate coordinated design of controllers for individual DFIG-based wind farms to collectively damp multiple critical oscillatory modes.

\section{DFIG-BASEd Wind FARM Modeling}

The overall structure of a DFIG is shown in Fig. 1 (a) where an aggregated model of the wind farm was adopted [1]. The objective of this work is to study the damping contribution from the wind farms. As the time-frame of power oscillation damping control study is less than half a minute the wind speed was assumed to remain constant during this interval. Also the turbine was assumed to operate in the zone of maximum power point extraction neglecting the pitch control.

Modeling of the DFIG was done in synchronously rotating $d-q$ reference frame [8] with $d$-axis leading the $q$-axis as per IEEE convention, see Fig. 1 (b). The stator transients of the machine were neglected, the converters were assumed to be ideal and the dc link dynamics was also neglected as suggested in [6] - further details can be found in [9]. Ideal frequency tracking was assumed neglecting the effect of phase locked loop (PLL) dynamics. Besides the standard differential and algebraic equations used to model the generator [9], a twomass model of the turbine and drive train was considered to take the torsional mode into account. The equations are not repeated here due to space restriction.

1) Rotor Side Converter (RSC) Control: Standard vector control approach [10] was adopted where the $q$-axis was aligned with $\psi_{s}$, see Fig. 1 (b). All notations in the modified reference frame are henceforth denoted with a prime. Therefore,

$$
\begin{aligned}
L_{s s} i_{d s}^{\prime}+L_{m} i_{d r}^{\prime}=0 & \Rightarrow i_{d s}^{\prime}=-\frac{L_{m}}{L_{s s}} i_{d r}^{\prime} \\
\psi_{q s}^{\prime} & =L_{s s} i_{q s}^{\prime}+L_{m} i_{q r}^{\prime}
\end{aligned}
$$
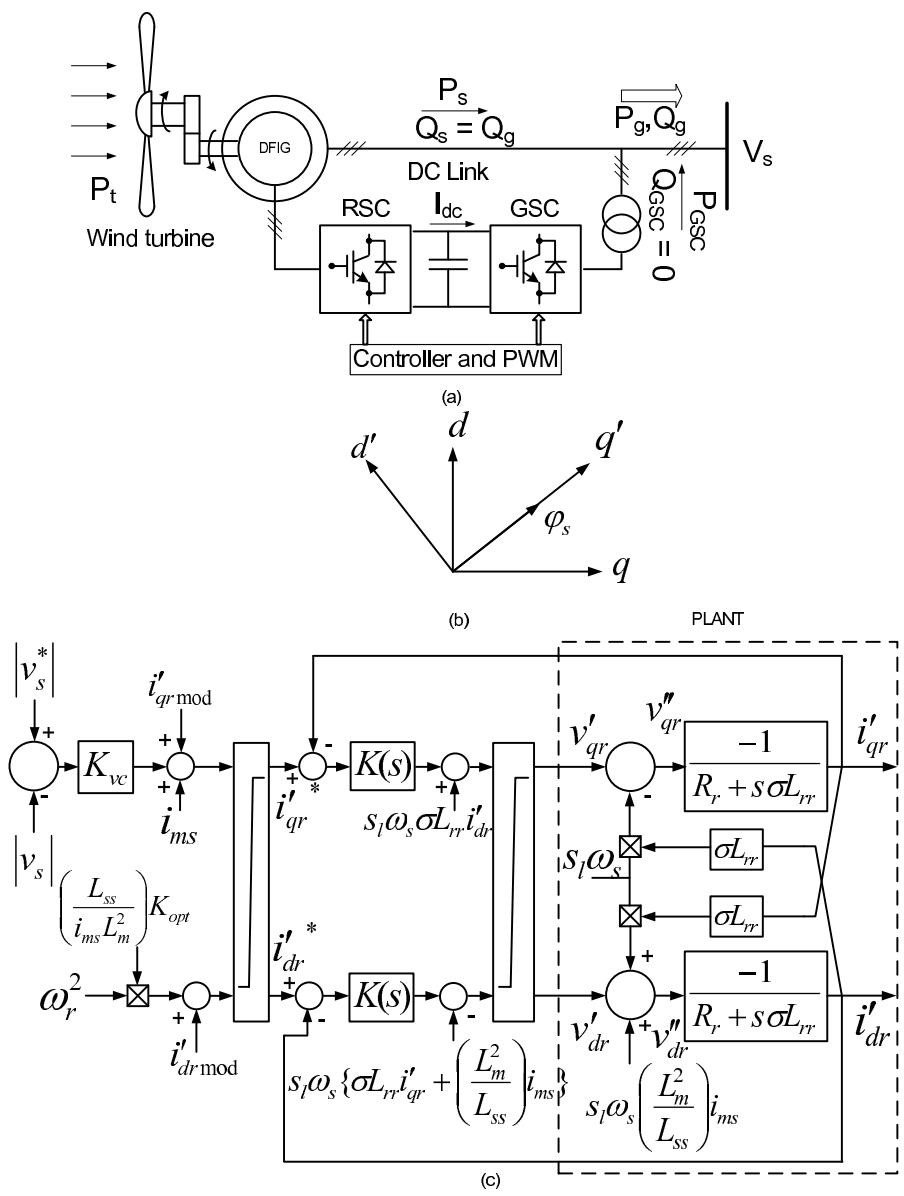

Fig. 1. (a) DFIG overall structure (b) $d-q$ : reference frame used for power system modeling, $d^{\prime}-q^{\prime}$ : modified reference frame for vector control (c) rotor converter control structure

Neglecting $R_{s}$ and assuming $i_{m s}$ constant we can write,

$$
\psi_{q s}^{\prime} \approx L_{m} i_{m s} \Rightarrow i_{q s}^{\prime}=\frac{L_{m}}{L_{s s}}\left(i_{m s}-i_{q r}^{\prime}\right)
$$

This results in simplification of $v_{d r}^{\prime}$ and $v_{q r}^{\prime}$ as follows:

$$
\begin{gathered}
v_{d r}^{\prime}=-R_{r} i_{d r}^{\prime}-\sigma L_{r r} \frac{d\left(i_{d r}^{\prime}\right)}{d t}-s_{l} \omega_{s} \sigma L_{r r} i_{q r}^{\prime}-s_{l} \omega_{s} \frac{L_{m}^{2}}{L_{s s}} i_{m s} \\
v_{q r}^{\prime}=-R_{r} i_{q r}^{\prime}-\sigma L_{r r} \frac{d\left(i_{q r}^{\prime}\right)}{d t}+s_{l} \omega_{s} \sigma L_{r r} i_{d r}^{\prime}
\end{gathered}
$$

where, $\sigma=\left(1-\frac{L_{m}^{2}}{L_{s s} L_{r r}}\right)$. As shown in Fig. 1 (c) 'PLANT', the above equations can be rewritten in terms of $v_{d r}^{\prime \prime}$ and $v_{q r}^{\prime \prime}$ after isolating the disturbance terms: $s_{l} \omega_{s} \sigma L_{r r} i_{d r}^{\prime}$ and $s_{l} \omega_{s}\left(\sigma L_{r r} i_{q r}^{\prime}+\frac{L_{m}}{L_{s s}} i_{m s}\right)$, respectively. Values of the parameters used for modelling are shown in Table I:

TABLE I

PARAMETERS USED FOR DFIG MODELING

\begin{tabular}{|c|c||c|c|}
\hline Parameters & Values $(\mathrm{pu})$ & Parameters & Values \\
\hline \hline$R_{s}$ & 0.00488 & $\omega_{s}$ & $1.0 \mathrm{pu}$ \\
\hline$L_{s s}$ & 4.0452 & $c_{s h}$ & 0.09 pu-s/elect. rad \\
\hline$L_{m}$ & 3.95279 & $k_{s h}$ & 0.3 pu/elect. rad \\
\hline$L_{r r}$ & 4.0523 & $H_{g}$ & $3.5 \mathrm{~s}$ \\
\hline$R_{r}$ & 0.00549 & $\omega_{\text {rrated }}$ & $1.3 \mathrm{pu}$ \\
\hline
\end{tabular}


Note that $i_{d r}^{\prime}$ and $i_{q r}^{\prime}$ are measurable parameters whereas $i_{m s}$ is estimated from (3). Therefore the measurable disturbances were used as feed-forward terms with appropriate signs to achieve decoupling between $d$ and $q$ axes current control loops. The torque reference was generated through Maximum power point tracking which in turn determined $i_{d r}^{\prime *}$ as shown below:

$$
T_{e}^{*}=\left(\frac{L_{m}^{2}}{L_{s s}}\right) i_{m s} i_{d r}^{\prime *}=K_{o p t} \omega_{r}^{2}
$$

$i_{q r}^{\prime *}$ was chosen so that the magnetizing current drawn by the induction generator is supplied through the RSC while injecting/absorbing appropriate reactive power depending on the difference between actual $\left(\left|V_{s}\right|\right)$ and reference $\left(\left|V_{s}^{*}\right|\right)$ voltage magnitude which in turn is controlled by $K_{v c}$.

Note that moderate closed loop bandwidth (BW) is adequate in tracking $i_{d r}^{*}$ and $i_{q r}^{\prime *}$ since they are dc in nature under steady state. Thus a BW of $300 \mathrm{rad} / \mathrm{s}$ was considered while designing the controller $K(s)$ as shown in the Table II.

TABLE II

DFIG PRIMARY CONTROLLER PARAMETERS

\begin{tabular}{|c|c|}
\hline Parameters & Values \\
\hline \hline$K(s)$ & $-300\left(\frac{R_{r}+s \sigma L_{r r}}{s}\right)$ \\
\hline$K_{v c}$ & 20.0 \\
\hline
\end{tabular}

2) Grid Side Converter (GSC) Control: The GSC was assumed to be lossless, i.e. the same real power flows through RSC and GSC. On the other hand $Q_{G S C}$ was kept zero to attain minimum converter size.

\section{TEST SYSTEM}

A 16-machine, 5-area system was considered for the case study, see Figs 2 and 3.

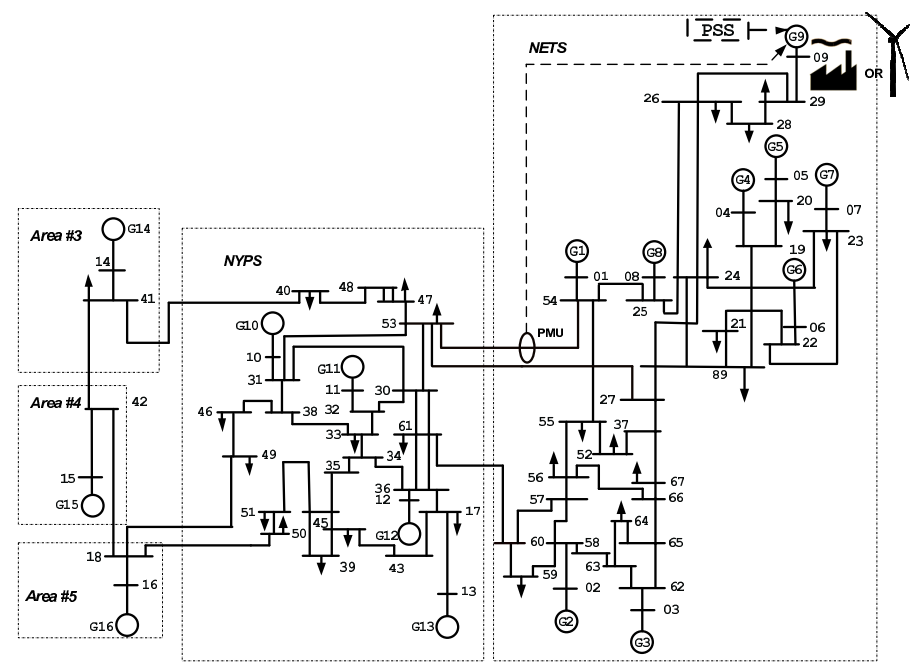

Fig. 2. Case Study 1: 16-machine, 5-area system where SG, G9 is equipped with a delta omega PSS. Impact of replacing this SG by a DFIG is studied. Remote feedback signal, $P_{54-53}$ is used for DFIG-PSS at $G 9$

All SGs were represented by sub-transient models and eight of them $(G 1-G 8)$ were equipped with IEEE DC1A excitation systems while a static excitation system with a PSS (referred to as SG-PSS in this paper for compactness) was installed

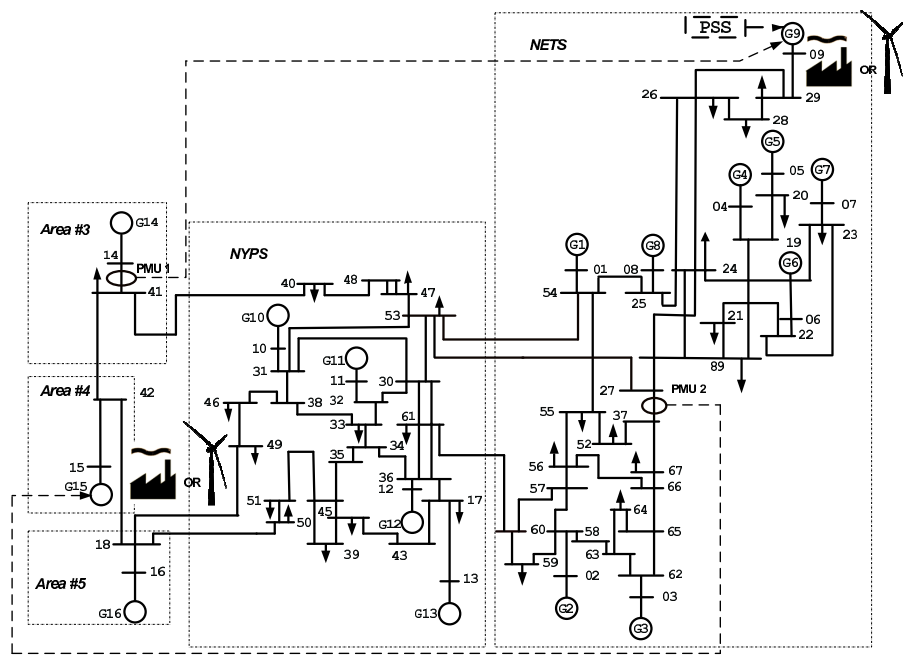

Fig. 3. Case Study 2: 16-machine, 5-area system where two SGs, $G 9$ and $G 15$ are replaced by DFIGs. Remote feedback signals, $P_{14-41}$ and $P_{27-37}$ are used for decentralized DFIG-PSSs at $G 9$ and $G 15$, respectively

at G9. The rest of the SGs were under manual excitation control. The active and reactive components of the loads had constant impedance characteristics. The SG-PSS structure and the corresponding parameters were taken from [11] as shown in the Appendix. The dynamic data for the system and nominal power transfer between areas can be found in [12]. Two scenarios were considered for case studies:

- Case Study 1: SG, G9 was replaced by a DFIG modeled as described earlier in Section II. The objective was to assess the impact on the system dynamics when a SG with PSS is replaced by a wind farm as shown in Fig. 2. Damping controller for the DFIG (referred to as DFIG-PSS henceforth) was designed to match the SGPSS performance

- Case Study 2: SGs, G9 and G15, were replaced by equivalent DFIGs, see Fig. 3. Coordinated design of the two DFIG PSSs in a decentralized framework was investigated

These two case studies are discussed in Sections V and VI.

In this context, it should be mentioned that the designed DFIG PSSs should modulate the control inputs of each wind turbine within a wind farm. In practice, the designed DFIGPSS will be installed at the central control center of the wind farm and the control command can be transmitted through fibre-optic interconnections to each turbine.

\section{Coordinated Control Design}

The objective was to ensure the following using a coordinated (simultaneous) approach to calculation of the parameters of multiple controllers:

1) A minimum settling time for all the closed-loop oscillatory modes under possible operating conditions

2) A diagonal controller resulting in decentralized control

3) Fixed structure low order controller

The coordinated control design problem was formulated as follows: 
Given a family of square linearized time-invariant (LTI) plants $G_{i}(s), i=1,2, \cdots, N$ corresponding to $N$ different operating conditions including the nominal one:

$G_{i}(s) \triangleq\left[\begin{array}{c|c}A_{i} & B_{i} \\ \hline C_{i} & 0\end{array}\right] ; A_{i} \in \Re^{n \times n}, B_{i} \in \Re^{n \times m}, C_{i} \in \Re^{m \times n}$

Find a stable diagonal LTI controller $K(s)$,

$$
\begin{aligned}
K(s) & \triangleq\left[\begin{array}{c|c}
A_{k} & B_{k} \\
\hline C_{k} & D_{k}
\end{array}\right] \\
& =\left[\begin{array}{cccc|cccc}
A_{k 1} & 0 & \cdot & 0 & B_{k 1} & 0 & \cdot & 0 \\
0 & A_{k 2} & \cdot & 0 & 0 & B_{k 2} & \cdot & 0 \\
. & \cdot & \cdot & \cdot & \cdot & \cdot & \cdot & . \\
0 & 0 & \cdot & A_{k m} & 0 & 0 & \cdot & B_{k m} \\
\hline C_{k 1} & 0 & \cdot & 0 & 0 & 0 & \cdot & 0 \\
0 & C_{k 2} & \cdot & 0 & 0 & 0 & \cdot & 0 \\
. & \cdot & \cdot & . & . & . & \cdot & . \\
0 & 0 & \cdot & C_{k m} & 0 & 0 & \cdot & 0
\end{array}\right]
\end{aligned}
$$

$A_{k j} \in \Re^{n_{k} \times n_{k}}, B_{k j} \in \Re^{n_{k} \times 1}, C_{k j} \in \Re^{1 \times n_{k}}, j=1,2, \cdots, m$ such that:

$$
\begin{aligned}
& K(s) \in \mathcal{S} \\
& \lambda\left(\left[\begin{array}{cc}
A_{i} & B_{i} C_{k} \\
B_{k} C_{i} & A_{k}
\end{array}\right]\right) \in \Im(\sigma) \quad \forall i=1,2, \cdots, N \\
& n_{k} \ll n
\end{aligned}
$$

where,

$\mathcal{S}$ : set of stable controllers

$\lambda(\cdot)$ : eigen values

$\Im(\sigma)$ : region to the left of constant $\sigma$ line specifying minimum settling time requirement.

Analytical solution to the above problem is not straightforward [13] due to its non-convex nature. Hence, heuristic optimization was used here to determine the PSS parameters embedded within $K(s)$ by solving the above problem. The optimization variables were the coefficients of the transfer functions describing the PSS. Constraint on closed-loop stability was implicitly imposed through introduction of a high penalty (e.g. $10^{6}$ ) in the objective function in case of closedloop poles on the right half of the s-plane.

To solve the problem using particle swarm optimization (PSO) technique, the solution space is initially populated with random numbers. Each potential solution, called a particle, is given a random velocity in $d$ dimension where, $\mathrm{d}$ is the number of PSS parameters to be optimized and flown through the problem space. Each particle is accelerated towards its individual best position and the global best position in each iteration. The optimal solution is obtained when the fitness of global best position is less than a pre-specified tolerance or the fitness does not change significantly over a number of consecutive iterations. For further details of the control design principle and methodology the readers can refer to [14], [15].

\section{CASe Study 1}

\section{A. Modal Analysis}

The test system was linearized around the nominal operating condition. Table III shows the damping ratios and frequencies of the electromechanical modes. There are four inter-area modes (referred to as mode \#1 to \#4 henceforth) with frequencies lying between $0.4-0.8 \mathrm{~Hz}$ and several local modes in the range of 1-2 Hz. When SG-PSS at $G 9$ is out of service, the damping of the $1^{\text {st }}$ and the $3^{\text {rd }}$ inter-area mode becomes poorer and the corresponding local mode with 1.19 $\mathrm{Hz}$ frequency becomes unstable whereas the eigenvalues of the other modes remain more or less constant, see Table III. Also note that the frequency of mode \#1 slightly increases due to absence of inertial contribution from the wind farm.

TABLE III

Damping Ratio and Frequency of Electromechanical Modes

\begin{tabular}{|c||c|c||c|c||c|c|}
\hline \multicolumn{1}{|c||}{ Scenarios } & \multicolumn{2}{|c||}{ SG with PSS } & \multicolumn{2}{c|}{ SG without PSS } & \multicolumn{2}{c|}{ DFIG } \\
\hline Modes & $\xi, \%$ & $f, H z$ & $\xi, \%$ & $f, H z$ & $\xi, \%$ & $f, H z$ \\
\hline \hline \multirow{3}{*}{$\begin{array}{c}\text { Inter- } \\
\text { Area }\end{array}$} & $\mathbf{6 . 5 0}$ & $\mathbf{0 . 3 8}$ & $\mathbf{1 . 6 0}$ & $\mathbf{0 . 3 9}$ & $\mathbf{1 . 5 0}$ & $\mathbf{0 . 4 0}$ \\
\cline { 2 - 7 } & 4.40 & 0.50 & 4.40 & 0.50 & 4.30 & 0.50 \\
\cline { 2 - 7 } & $\mathbf{5 . 7 0}$ & $\mathbf{0 . 6 2}$ & $\mathbf{3 . 6 0}$ & $\mathbf{0 . 6 3}$ & $\mathbf{4 . 5 0}$ & $\mathbf{0 . 6 2}$ \\
\cline { 2 - 7 } & 5.00 & 0.79 & 5.00 & 0.79 & 5.00 & 0.79 \\
\hline \hline \multirow{5}{*}{ Local } & 6.30 & 1.07 & 6.30 & 1.07 & 6.30 & 1.07 \\
\cline { 2 - 7 } & 5.90 & 1.16 & 5.80 & 1.16 & 5.90 & 1.16 \\
\cline { 2 - 7 } & 8.00 & 1.21 & 7.90 & 1.21 & 8.00 & 1.20 \\
\cline { 2 - 7 } & $\mathbf{5 . 8 0}$ & $\mathbf{1 . 2 6}$ & $\mathbf{- 0 . 4 0}$ & $\mathbf{1 . 1 9}$ & - & - \\
\cline { 2 - 7 } & 7.50 & 1.27 & 7.40 & 1.27 & 7.40 & 1.27 \\
\cline { 2 - 7 } & 5.00 & 1.34 & 3.50 & 1.35 & 4.70 & 1.34 \\
\cline { 2 - 7 } & 9.80 & 1.54 & 9.80 & 1.54 & 9.80 & 1.54 \\
\cline { 2 - 7 } & 6.80 & 1.55 & 6.90 & 1.55 & 6.80 & 1.55 \\
\cline { 2 - 7 } & 9.10 & 1.56 & 9.10 & 1.56 & 9.10 & 1.56 \\
\cline { 2 - 7 } & 6.20 & 1.88 & 6.20 & 1.88 & 6.20 & 1.88 \\
\hline
\end{tabular}

The impact of replacing the SG, G9 with a DFIG was similar to that of keeping the PSS out of service - note similar effect on the inter-area modes. However, the major difference was that the unstable $1.19 \mathrm{~Hz}$ local mode was absent due to the 'decoupling' effect of DFIG at $G 9$.

Fig. 4 shows the relative mode shapes of generator speeds for mode \#1 to \#4 for the case with SG-PSS vis-a-vis DFIG at $G 9$. For sake of clarity, generators with highest participation from each area are shown along with $G 9$. Note that $G 9$ does not show up in the mode shape when it is a DFIG (see right hand column of Fig. 4) unlike when it is SG (see left hand column of Fig. 4). The DFIG-PSS might not be effective in damping the inter-area modes, since the mechanical states of DFIGs do not participate in them. Miller et-al [1] showed that the DFIGs do not contribute to electromechanical modes and as a result the PSS action is not needed in them [7]. On the other hand, Jenkins et-al [16] and Vournas et-al [2] showed the damping potential of DFIGs using mainly local feedback signals (e.g. $\omega_{r}, P_{\text {stator }}$ etc.) while Fan et-al [3] used a remote feedback signal. The damping potential of wind farms and related control loop design is discussed in the following section.

\section{B. Control Loop}

As shown in Table III, introduction of DFIG, G9 reduces the damping of two inter-area modes necessitating PSS action. Since the RSC of DFIG was controlled based on current control strategy, see Section II-1, the $d$ and $q$ axes rotor currents were chosen for modulation. Modal controllability of the rotor currents, shown in Table IV, has non-zero magnitudes 


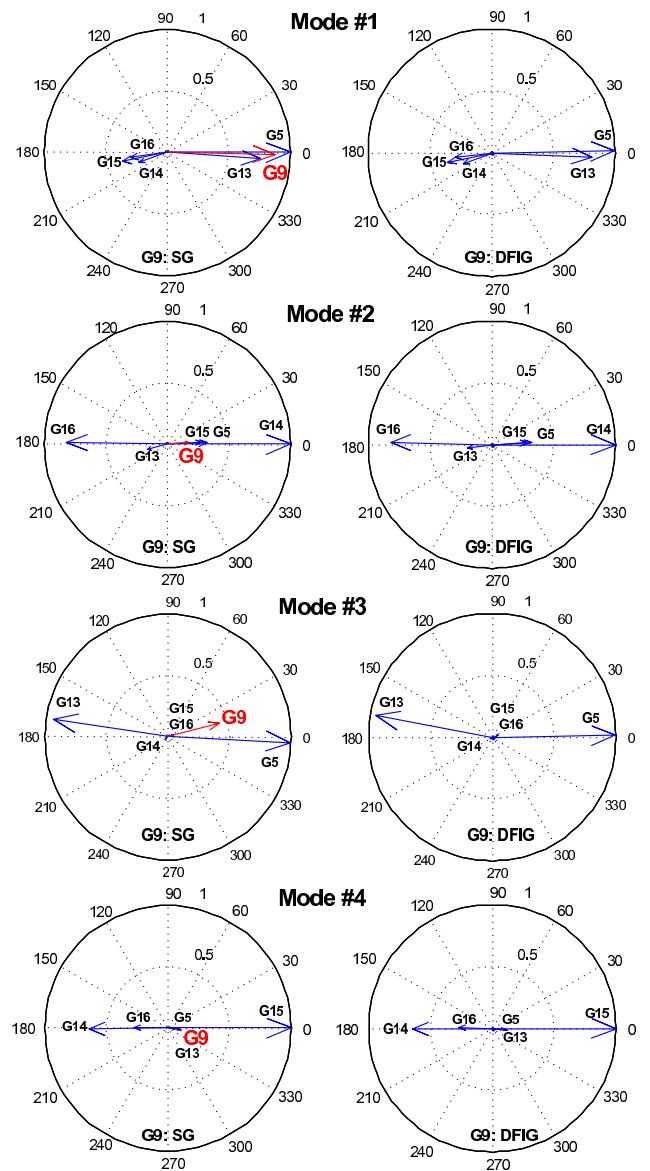

Fig. 4. Relative mode shapes of generator speeds for four inter-area modes where $G 9$ (Red/ Grey arrow) is either SG or DFIG. Generators from each area with highest participation is shown. DFIG, $G 9$ has negligible participation in inter-area modes (Red/Grey arrows not visible in right half column)

which confirms that DFIG-PSS has the potential to damp interarea oscillations. For all four modes, $I_{d r m o d}^{\prime}$ is seen to offer higher controllability than $I_{\text {qrmod }}^{\prime}$.

TABLE IV

Modal CONTROLlability OF DFIG Rotor CURRENTS

\begin{tabular}{|c||c|c|c|c|}
\hline Mode & $\# 1$ & $\# 2$ & $\# 3$ & $\# 4$ \\
\hline \hline$I_{\text {drmod }}^{\prime}$ & 0.762 & 0.021 & 0.696 & 0.006 \\
\hline$I_{\text {qrmod }}^{\prime}$ & 0.553 & 0.015 & 0.506 & 0.004 \\
\hline
\end{tabular}

The objective here was to match the SG-PSS performance through DFIG-PSS by modulation of rotor currents. Modal observability of power flow signals were calculated and compared against the SG-PSS case. Table V shows the normalized observability for a few line real power flows where $P_{13-17}$ has maximum observability for mode $\# 1$ and $\# 3, P_{16-18}$ for mode \#2 and $P_{15-42}$ for mode \#3. The observability of all four modes in the DFIG stator power $P_{G 9}$ is significantly less as compared to the case with SG, thereby, ruling this out as an effective feedback signal. Other signals available at the DFIG location including DFIG bus voltage and frequency have much less modal energy compared to stator power and were therefore, not considered.

Residue angle criteria [17] was used for short listing the signals which were then arranged in the descending order
TABLE V

Modal Observability of Local and Remote Power Flow Signals

\begin{tabular}{|c||c|c||c|c||c|c||c|c|}
\hline \multicolumn{1}{|c|}{ Mode } & \multicolumn{2}{|c||}{$\# 1$} & \multicolumn{2}{|c|}{$\# 2$} & \multicolumn{2}{|c|}{$\# 3$} & \multicolumn{2}{|c|}{$\# 4$} \\
\hline Signals & SG-PSS & DFIG & SG-PSS & DFIG & SG-PSS & DFIG & SG-PSS & DFIG \\
\hline \hline$P_{G 9}$ & $\mathbf{0 . 0 7 7}$ & $\mathbf{0 . 0 0 3}$ & $\mathbf{0 . 0 1 6}$ & $\mathbf{0 . 0 0 1}$ & $\mathbf{0 . 0 3 1}$ & $\mathbf{0 . 0 0 1}$ & $\mathbf{0 . 0 0 6}$ & $\mathbf{0 . 0 0 0}$ \\
\hline$P_{54-53}$ & 0.381 & 0.309 & 0.141 & 0.146 & 0.283 & 0.278 & 0.033 & 0.034 \\
\hline$P_{37-68}$ & 0.223 & 0.216 & 0.100 & 0.107 & 0.211 & 0.216 & 0.026 & 0.032 \\
\hline$P_{61-60}$ & 0.268 & 0.211 & 0.196 & 0.204 & 0.334 & 0.332 & 0.013 & 0.016 \\
\hline$P_{19-68}$ & 0.156 & 0.143 & 0.073 & 0.076 & 0.148 & 0.148 & 0.018 & 0.021 \\
\hline$P_{55-54}$ & 0.162 & 0.142 & 0.060 & 0.066 & 0.139 & 0.142 & 0.020 & 0.022 \\
\hline$P_{52-55}$ & 0.152 & 0.120 & 0.079 & 0.083 & 0.154 & 0.153 & 0.015 & 0.017 \\
\hline$P_{52-37}$ & 0.150 & 0.119 & 0.072 & 0.075 & 0.142 & 0.140 & 0.016 & 0.017 \\
\hline$P_{13-17}$ & 1.000 & 1.000 & 0.238 & 0.279 & 1.000 & 1.000 & 0.157 & 0.151 \\
\hline$P_{16-18}$ & 0.351 & 0.317 & 1.000 & 1.000 & 0.045 & 0.046 & 0.412 & 0.412 \\
\hline$P_{15-42}$ & 0.284 & 0.260 & 0.229 & 0.235 & 0.008 & 0.008 & 1.000 & 1.000 \\
\hline
\end{tabular}

of observability of mode \#1, see Table V. A single-input multiple-output controller was used with $P_{54-53}$ as the feedback signal (see Fig. 2) and both $d$ and $q$ axes rotor currents with comparable modal controllability as control inputs. Control design approach outlined in Section IV was adopted to achieve closed loop settling times similar to that with a SG-PSS with $n_{k}=2$, see (8). As shown in Fig. 6, the closed loop settling times of the DFIG-PSS is comparable to that of SG-PSS with mode \#3 being better damped in the former case. This figure also incorporates the previous observations regarding settling times of inter-area modes for ease of understanding. The controller structure is shown in Fig. 5.

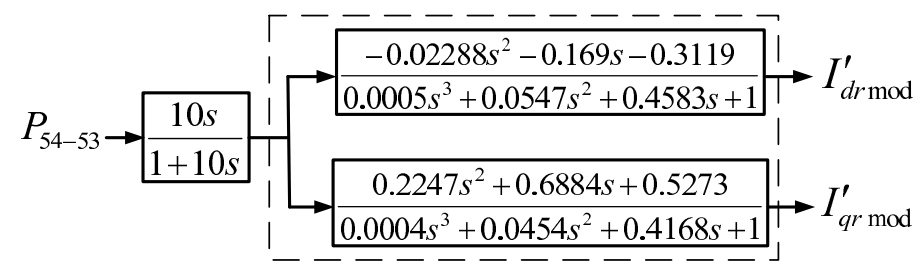

Fig. 5. Parameters of the PSS for DFIG, G9

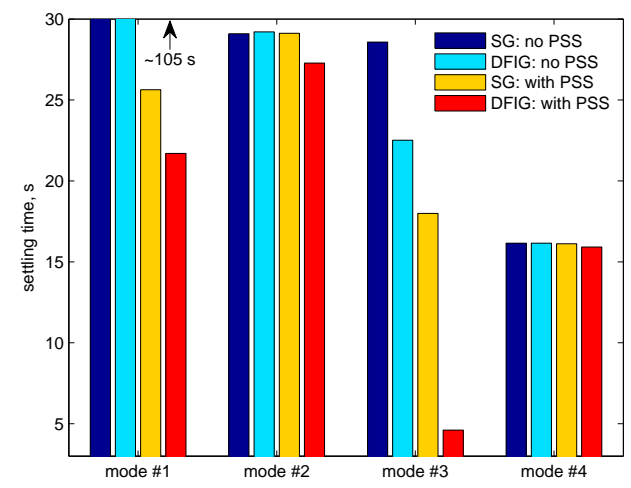

Fig. 6. Settling times of four inter-area modes with (a) SG, $G 9$ without any PSS (b) DFIG, G9 without PSS (c) SG, G9 with PSS and (d) DFIG, G9 with PSS to match/improve over SG-PSS performance

Note that, for the test system used in this paper, only G9 was equipped with PSS. However, the formulation in Section IV is general for any multi-input multi-output system and hence applicable to cases where more than one SG-PSS is involved. 


\section{Simulation Results}

Non-linear simulation was done in MATLAB/SIMULINK to confirm the findings of the linear analysis and figure out whether DFIG-PSS can match the SG-PSS performance. Fig. 7 shows the system behavior following a three-phase selfclearing fault near bus 60 for $80 \mathrm{~ms}$.
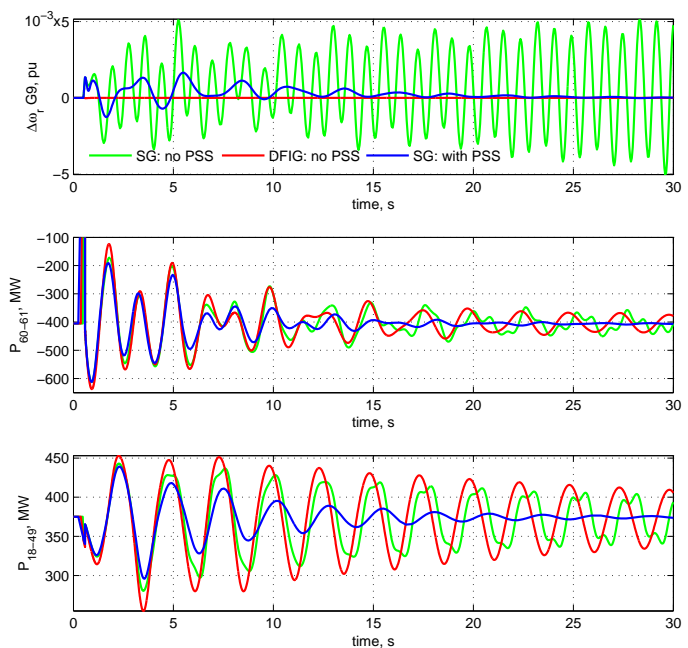

Fig. 7. Dynamic performance of the system (see Fig. 2) following a selfclearing 3-phase fault near bus 60 for $80 \mathrm{~ms}$. Green/Light Grey trace: SG, $G 9$ without PSS - a local mode is unstable. Red/Dark Grey trace: DFIG, G9 without PSS. Blue/Black trace: SG, $G 9$ with PSS

When the PSS at SG, G9 is taken out of service, instability of the local mode is seen in the speed deviation of $G 9$ and also in tie-power flows to a lesser extent. The presence of SG-PSS shows its stabilizing effect on the system whereas replacement of $G 9$ by DFIG results in poorly damped interarea oscillations. Speed deviation of the DFIG shows negligible oscillations which confirms the mode-shapes of generator speed in Fig. 4. However, if the fault location is electrically closer to the DFIG bus, situation could be different.

Effectiveness of the DFIG-PSS is illustrated in Fig. 8 against that of DFIG with only primary controls and SG-PSS scenario.

It is interesting to note that inter-area mode is observable in $\Delta \omega_{r}$ and $\theta_{t w}$ when supplementary control is employed, thereby, obtaining similar damping performance like the SGPSS.

The variation of the DFIG bus voltage and reactive power injection/absorption with and without PSS is shown in Fig 9.

Note that, the reference voltage $\left(\left|V_{s}^{*}\right|\right)$ was initialized to its actual value $\left(\left|V_{s}\right|\right)$ based on the load flow solution. Therefore, the steady state offset (about 0 ) in reactive power (see zoomed view) is the contribution towards the magnetizing component. Looking at the scenario with no PSS (red traces), it is clear when $\left|V_{s}\right|>\left|V_{s}^{*}\right|$ the DFIG draws negative reactive power (acts like an inductor) and vice versa. The reactive power injection/absorption depends on the difference between actual and reference voltage magnitudes. However, with PSS (blue trace) a modulating component is added to the current control loop which disrupts the above mentioned phase relationship.
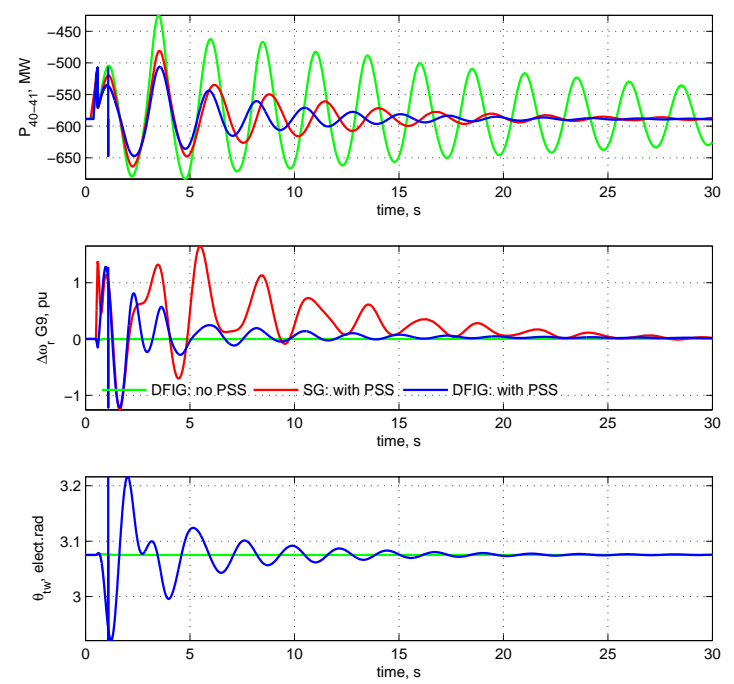

Fig. 8. Dynamic performance of the system following a self-clearing 3-phase fault near bus 60 for $80 \mathrm{~ms}$. Green/Light Grey trace: DFIG, G9 without PSS. Red/Dark Grey trace: SG, G9 with PSS. Blue/Black trace: DFIG, G9 with PSS to match/improve SG-PSS performance
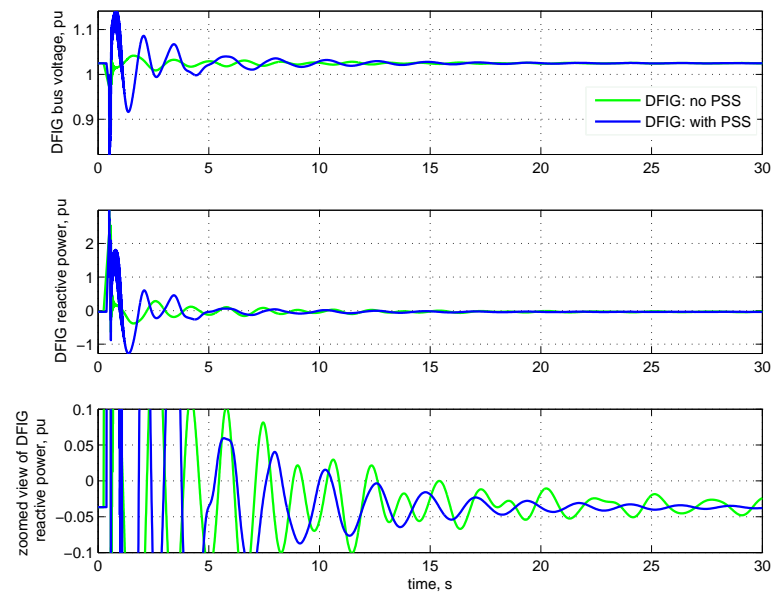

Fig. 9. Dynamic variation of DFIG bus voltage and reactive power following a self-clearing 3-phase fault near bus 60 for $80 \mathrm{~ms}$. Green/Light Grey trace: DFIG, G9 without PSS. Blue/Black trace: DFIG, G9 with PSS

\section{Case Study 2}

In this section coordinated control of multiple DFIGs in a decentralized framework is discussed for the system shown in Fig. 3, where in addition to $G 9$, the $\mathrm{SG}$ at $G 15$ was also replaced by an equivalent DFIG. Modal analysis and controller design were done to achieve a settling time of $15.0 \mathrm{~s}$.

\section{A. Modal Analysis}

Linear analysis around a nominal condition shows the presence of only three dominant inter-area modes, whose settling times are shown in Fig. 12. As shown in Fig. 4, SG at G15 had the highest participation in mode \#4 and the rest of the system was oscillating against it. When this generator was replaced by a DFIG, the corresponding inter-area mode ceased to exist. Mode shape plots in Fig. 10 illustrates the lack of participation 
of $G 9$ and $G 15$ (DFIGs) speeds in all the three modes. Closer look reveals that the relative mode shape magnitude of $G 5$ and G16 reduces in mode \#2 and that of G16 increases slightly in mode \#1 for the DFIG scenario.
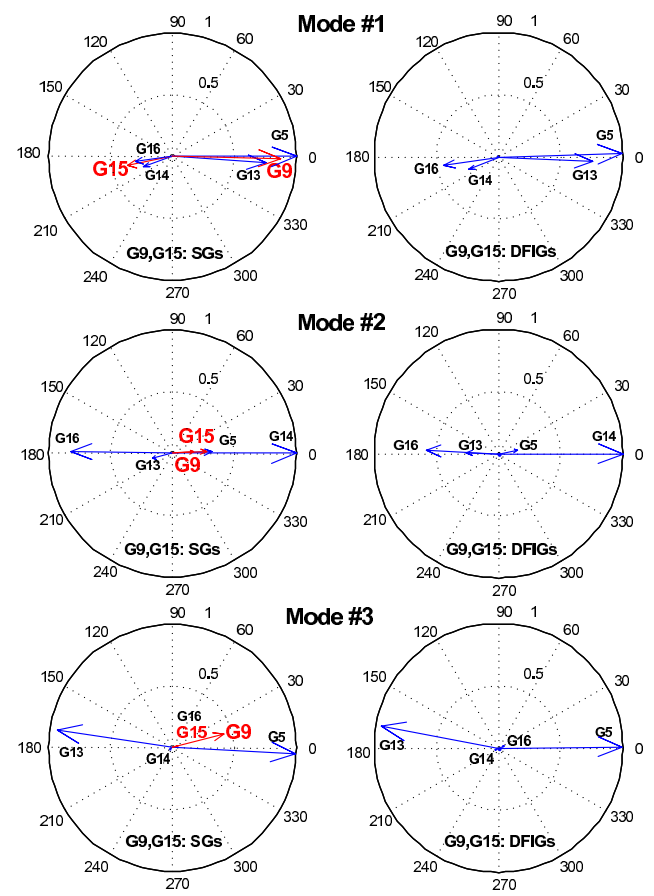

Fig. 10. Relative mode shapes of generator speeds for three inter-area modes where $G 9$ and $G 15$ (Red/ Grey arrow) are either SGs or DFIGs. Generators from each area with highest participation is shown. When $G 9$ and $G 15$ are DFIGs they have negligible participation in inter-area modes. When $G 15$ is DFIG the fourth mode is absent

\section{B. Control Loop}

Modal controllability of DFIG rotor currents for $G 9$ and G15 shows that $I_{d r m o d}^{\prime}$ of $G 9$ is the natural choice for modulation as this has the highest magnitudes for mode \#1 and \#3, see Table VI. Additionally, $I_{q r m o d}^{\prime}$ of $G 15$ was selected, in spite of a higher controllability of $G 9 q$-axis rotor current for mode \#2 (see Table VI) in order to distribute the damping duty amongst the available wind farms. The rule of selection of the control inputs for multiple DFIG-PSSs should be as follows:

1) For each mode, arrange the control inputs of the DFIGs in a descending order of controllability.

2) Select the control input based on the following parameters: 1) higher controllability and 2) damping duty

TABLE VI

Modal Controllability of DFIG Rotor Currents

\begin{tabular}{|c||c|c|c|c|}
\hline \multicolumn{2}{|c|}{ Mode } & $\# 1$ & $\# 2$ & $\# 3$ \\
\hline \hline \multirow{2}{*}{$G 9$} & $I_{\text {drmod }}^{\prime}$ & 0.639 & 0.025 & 0.694 \\
\cline { 2 - 5 } & $I_{\text {qrmod }}^{\prime}$ & 0.322 & 0.090 & 0.010 \\
\hline \multirow{2}{*}{ G15 } & $I_{\text {drmod }}^{\prime}$ & 0.464 & 0.018 & 0.505 \\
\cline { 2 - 5 } & $I_{\text {qrmod }}^{\prime}$ & 0.194 & 0.040 & 0.011 \\
\hline
\end{tabular}

As shown in Table VII, magnitudes of modal observability of stator powers of $G 9$ and $G 15$ are significantly reduced when corresponding SGs are replaced by DFIGs.
TABLE VII

Modal Observability of Local and Remote Power Flow Signals

\begin{tabular}{|c||r|r||r|r||r|r|}
\hline \multicolumn{1}{|c||}{ Mode } & \multicolumn{2}{c||}{$\# 1$} & \multicolumn{2}{c||}{$\# 2$} & \multicolumn{2}{c|}{$\# 3$} \\
\hline Signals & SG & DFIG & SG & DFIG & SG & DFIG \\
\hline \hline$P_{G 9}$ & $\mathbf{0 . 0 7 7}$ & $\mathbf{0 . 0 0 3}$ & $\mathbf{0 . 0 1 6}$ & $\mathbf{0 . 0 0 0}$ & $\mathbf{0 . 0 3 1}$ & $\mathbf{0 . 0 0 1}$ \\
\hline$P_{G 15}$ & $\mathbf{0 . 2 8 4}$ & $\mathbf{0 . 0 0 2}$ & $\mathbf{0 . 2 2 9}$ & $\mathbf{0 . 0 0 1}$ & $\mathbf{0 . 0 0 8}$ & $\mathbf{0 . 0 0 0}$ \\
\hline$P_{14-41}$ & 0.192 & 0.197 & 0.813 & 1.000 & 0.015 & 0.016 \\
\hline$P_{27-37}$ & 0.072 & 0.102 & 0.028 & 0.017 & 0.069 & 0.075 \\
\hline$P_{13-17}$ & 1.000 & 1.000 & 0.238 & 0.453 & 1.000 & 1.000 \\
\hline
\end{tabular}

Based on residue angle criterion mentioned in the previous section, $P_{14-41}$ and $P_{27-37}$ were chosen as feedback signals for $G 9$ and $G 15$, respectively, see Fig. 3. The decentralized controllers were designed with $n_{k}=3$, see (8), and their parameters are shown in Fig. 11.

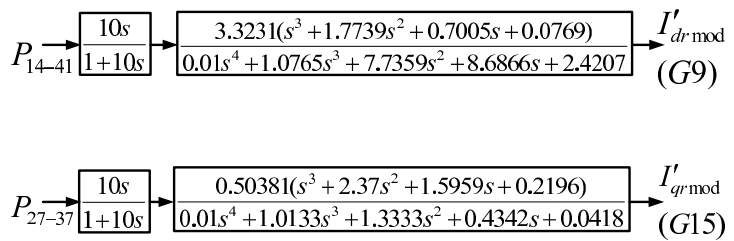

Fig. 11. Parameters of the PSSs for DFIGs, $G 9$ and $G 15$

It can be seen from Fig. 12 that a settling time of about $15.0 \mathrm{~s}$ or less was achieved in closed loop for all three modes as imposed by the design criterion (IV).

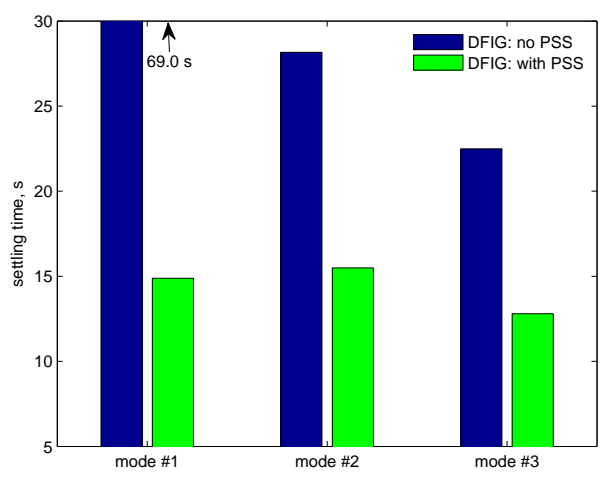

Fig. 12. Settling times of three inter-area modes with DFIGs, $G 9$ and $G 15$ without/ with PSSs

\section{Simulation Results}

The dynamic performance of the system following a threephase self-clearing fault of 5-cycle duration near bus 60 is shown in Fig. 13.

The real power flow in the tie-line 54-53 connecting NETS and NYPS shows the presence of three poorly damped modes for DFIGs without PSSs. Modulation of $I_{d r}^{\prime}$ of $G 9$ and $I_{q r}^{\prime}$ of $G 15$ resulted in settling of these modes in about $15.0 \mathrm{~s}$. The amplitude of modulation of $I_{d r}^{\prime}$ is higher as compared to that of $I_{q r}^{\prime}$. Note that the DFIG rotor current components are expressed in the modified reference frame as shown in Fig. 1 (b) whereby the $d^{\prime}$ axes are locked with respect to the stator flux of individual generators $G 9$ and $G 15$. 

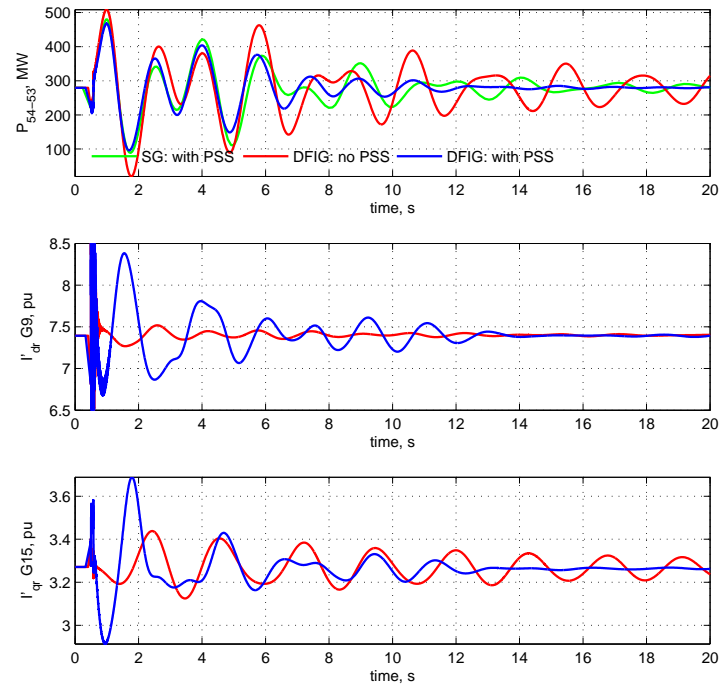

Fig. 13. Dynamic performance of the system (see Fig. 3) following a selfclearing 3-phase fault near bus 60 for $80 \mathrm{~ms}$. Green/Light Grey trace: SGs, $G 9$ and $G 15$. Red/Dark Grey trace: DFIGs, $G 9$ and $G 15$ without PSSs. Blue/Black trace: DFIGs, $G 9$ and $G 15$ with PSSs
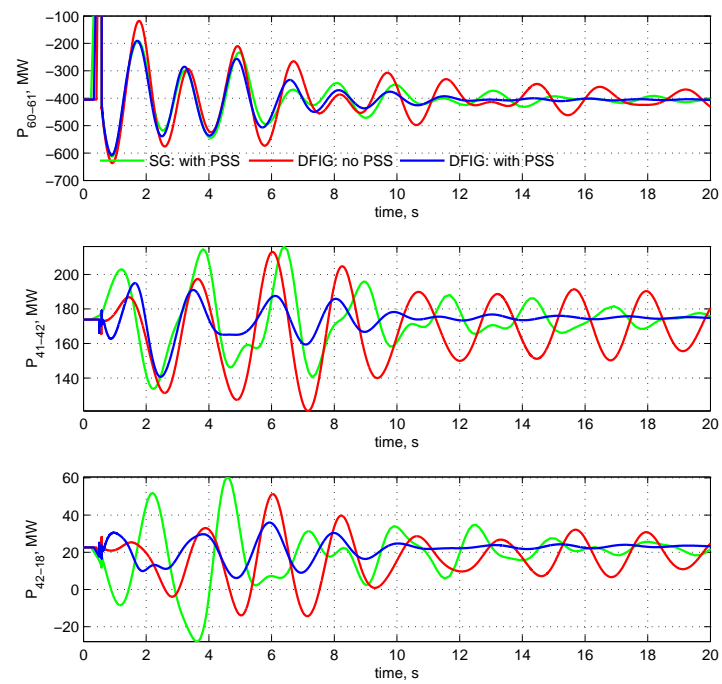

Fig. 14. Dynamic performance of the system (see Fig. 3) following a selfclearing 3-phase fault near bus 60 for $80 \mathrm{~ms}$. Green/Light Grey trace: SGs, $G 9$ and $G 15$. Red/Dark Grey trace: DFIGs, $G 9$ and $G 15$ without PSSs. Blue/Black trace: DFIGs, $G 9$ and $G 15$ with PSSs

Fig. 14 shows the real power flows through the three tielines connecting different areas. The power flow through the line connecting buses 60 and 61 suffers the worst dip due to its proximity to the fault location. The dynamic performance of the system illustrates the effectiveness of the decentralized PSSs at two distant wind farms in damping the three critical modes.

\section{CONCLUSION}

Important considerations towards coordinated control of doubly-fed induction generator (DFIG) based wind farms for power oscillation damping were analyzed. The design methodology and performance validation has been demonstrated through modal analysis and non-linear simulations.

\section{APPENDIX}

Parameters of the PSS at SG, G9 are shown below:

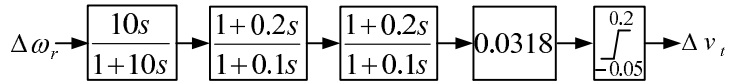

Fig. 15. Parameters of SG-PSS at G9 (from [11])

\section{REFERENCES}

[1] J. J. Sanchez-Gasca, N. W. Miller, and W. W. Price, "A modal analysis of a two-area system with significant wind power penetration," in IEEE PES Power Systems Conference and Exposition, 2004., 2004, pp. 11481152 vol. 2 .

[2] G. Tsourakis, B. M. Nomikos, and C. D. Vournas, "Contribution of doubly fed wind generators to oscillation damping," IEEE Transactions on Energy Conversion, vol. 24, no. 3, pp. 783-791, 2009.

[3] M. Zhixin, F. Lingling, D. Osborn, and S. Yuvarajan, "Control of dfigbased wind generation to improve interarea oscillation damping," IEEE Transactions on Energy Conversion, vol. 24, no. 2, pp. 415-422, 2009.

[4] E. Hagstrm, I. Norheim, and K. Uhlen, "Large-scale wind power integration in norway and impact on damping in the nordic grid," Wind Energy, vol. 8, no. 3, pp. 375-384, 2005.

[5] D. J. Vowles, C. Samarasinghe, M. J. Gibbard, and G. Ancell, "Effect of wind generation on small-signal stability; a new zealand example," in proceedings of IEEE Power and Energy Society General Meeting, 2008, pp. 1-8.

[6] F. M. Hughes, O. Anaya-Lara, N. Jenkins, and G. Strbac, "Control of dfig-based wind generation for power network support," IEEE Transactions on Power Systems, vol. 20, no. 4, pp. 1958-1966, 2005.

[7] R. Piwko, N. Miller, R. Girad, J. MacDowell, K. Clark, and A. Murdoch, "Generator fault tolerance and grid codes," IEEE Power and Energy Magazine, vol. 8, no. 2, pp. 18-26, 2010.

[8] P. Kundur, Power system stability and control, ser. The EPRI power system engineering series. New York; London: McGraw-Hill, 1994.

[9] J. G. Slootweg, H. Polinder, and W. L. Kling, "Dynamic modelling of a wind turbine with doubly fed induction generator," in IEEE Power Engineering Society Summer Meeting, 2001, vol. 1, 2001, pp. 644-649 vol.1.

[10] R. Pena, J. C. Clare, and G. M. Asher, "Doubly fed induction generator using back-to-back pwm converters and its application to variablespeed wind-energy generation," IEE Proceedings on Electric Power Applications, vol. 143, no. 3, pp. 231-241, 1996.

[11] G. Rogers, Power system oscillations. Boston; London: Kluwer Academic, 2000.

[12] B. Pal and B. Chaudhuri, Robust control in power systems, ser. Power electronics and power systems. New York: Springer, 2005.

[13] S. P. Bhattacharyya, H. Chapellat, and L. H. Keel, Robust control : the parametric approach. Upper Saddle River London ;: Prentice Hall ; Prentice-Hall International, 1995

[14] J. Kennedy and R. Eberhart, "Particle swarm optimization," in IEEE International Conference on Neural Networks, vol. 4, 1995, pp. 19421948.

[15] B. Chaudhuri, S. Ray, and R. Majumder, "Robust low-order controller design for multi-modal power oscillation damping using flexible $\mathrm{AC}$ transmission systems devices," IET Generation, Transmission \& Distribution, vol. 3, no. 5, pp. 448-459, 2009.

[16] F. M. Hughes, O. Anaya-Lara, N. Jenkins, and G. Strbac, "A power system stabilizer for dfig-based wind generation," IEEE Transactions on Power Systems, vol. 21, no. 2, pp. 763-772, 2006.

[17] S. Ray, B. Chaudhuri, and R. Majumder, "Appropriate signal selection for damping multi-modal oscillations using low order controllers," in proceedings of IEEE Power Engineering Society General Meeting, 2008, Pittsburgh, 2008. 


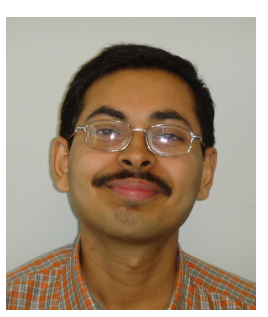

Nilanjan Ray Chaudhuri (S'08-M'09) received his $\mathrm{Ph} . \mathrm{D}$. in electrical power systems from Imperial College London in 2011. His research interests include power system dynamics and control, application of power electronics in power systems, online system identification, FACTS, HVDC and renewable energy systems.

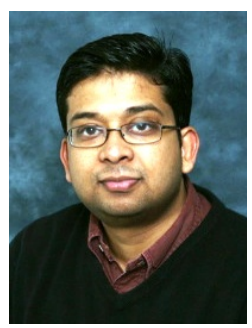

Balarko Chaudhuri (S'02-M'06-SM'11) received the Ph.D. degree in 2005 from Imperial College London, U.K. He is presently a Senior Lecturer in the Control and Power Research Group, Imperial College London. His area of interest is electric power transmission systems, control theory, smart grids and renewable energy. 